\title{
VAT Compliance: Einhaltung umsatzsteuerlicher Anforderungen beim Prozess- und Datenmanagement in ERP-Systemen
}

\author{
Nick Gebrke1, Rainer Thams² \\ ${ }^{1}$ Arbeitsbereich Wirtschaftsinformatik, Universität Hamburg \\ 2PricewaterhouseCoopers $A G$, Hamburg
}

\section{Einleitung}

Der vorliegende Beitrag beschäftigt sich mit dem Thema, wie umsatzsteuerliche Anforderungen, vorgegeben vom Umsatzsteuergesetz, bei der Abwicklung von Geschäftsvorfällen in ERP-Systemen ordnungsgemäß berücksichtigt werden können. Es handelt sich um ein Compliance Thema, da diese spezifischen Anforderungen der Gesetzgebung entspringen. Der Beitrag fokussiert dabei zwei Szenarien: erstens die Sicherstellung der umsatzsteuerlichen Ordnungsmäßigkeit bei der Einführung von ERP-Systemen und zweitens eine Betrachtung im Regelbetrieb mit Hilfe eines sogenannten VAT-Scans.

Der Beitrag ist wie folgt gegliedert. Zunächst wird der Stand der Forschung dargelegt. Dabei stellt sich heraus, dass bisher wenig wissenschaftliche Literatur zu diesem Thema vorliegt, jedoch bereits Arbeiten dazu existieren, wie rechtliche Bestimmungen in Anforderungen an IT-Systeme überführt werden können. Anschließend folgt die Darlegung der Problemrelevanz. Da das Thema Bezüge zum Recht als auch zur IT hat, werden folgend Grundsätze des Umsatzsteuerrechts erläutert. Aufgrund der Rechtskomplexität kann dies nur oberflächlich geschehen. Danach werden Vorgehensweisen dargestellt, wie Anforderungen des Umsatzsteuerrechts bei der Konzeption der Einführung eines ERP-Systems berücksichtigt werden können. Zwei verschiedene Vorgehensweisen werden an dieser Stelle dargestellt. Anschließend wird eine Vorgehensweise skizziert, wie im laufenden Betrieb eines ERP-Systems die Einhaltung der umsatzsteuerlichen Bestimmungen beurteilt werden kann. Der Beitrag schließt mit einem kurzen Fazit. 


\section{Stand der Forschung}

Im Rahmen der Entwicklung und Einführung von IT-Systemen beschäftigt sich die Disziplin des Requirements Engineerings mit der Erhebung und Umsetzung von Anforderungen in IT-Systemen (Nuseibeh und Easterbrook 2000). Gesetzliche Anforderungen können als eine bestimmte Art von Anforderungen angesehen werden, die auch im Rahmen des Requirements Engineering erfasst werden müssen. Wie aus Gesetzen Anforderungen extrahiert werden können, um Anforderungen an IT-Systeme abzuleiten, wird dabei verstärkt erst in jüngerer Zeit in der wissenschaftlichen Literatur diskutiert. Im Mittelpunkt stehen hier jedoch weniger Steuergesetze als eher z. B. Datenschutzgesetze (Privacy Act) oder auch spezielle Gesetze wie der Health Insurance Portability and Accountability Act (HIPAA) oder der Sarbanes Oxley Act (SOX) in den USA (Biagioli et a. 2005, Breaux et al. 2006, Breaux et al. 2008a, Breaux et al. 2008b, Ghanavati et al. 2007, Karagiannis 2008, Kiavitskaya et al. 2008, Kiyavitskaya et al. 2008, Kiyavitskaya et al. 2007, Otto und Anton 2007, Palmirani und Massini 2003, Siena und Mylopoulos 2008). Als eine der wenigen Beiträge, die sich Explizit mit umsatzsteuerlichen Problemen im Zusammenhang mit ERP-Systemen beschäftigen sind Goossenaerts et al. (2009) zu nennen, die ein modellgetriebenes Regelwerk für VAT-Compliance in ERP-Systemen entwickeln. Im deutschsprachigen Raum scheint das Thema bisher nahezu unbearbeitet. In der Revisionsliteratur im Bereich der elektronischen Prüfung von steuerrelevanten Daten mit Hilfe von spezieller Prüfsoftware ist hauptsächlich praxisrelevante Literatur zu finden (Maliszewski 2008, Odenthal 2006). Auf dem Softwaremarkt gibt es einige wenige Nischenanbieter, die explizit auf die Prüfung von umsatzsteuerlichen Sachverhalten fokussieren (zu nennen wären hier SAP Tax Audit for SAP ERP, ESKORT (Toolbox for VAT auditing) und Vertex Valued Added Tax Series).

\section{Problemrelevanz}

Die Problemrelevanz von VAT Compliance im Zusammenhang mit Geschäftsprozessen in ERP-Systemen ergibt sich hauptsächlich aus der Komplexität des europäischen Umsatzsteurrechts und den damit verbundenen Problemen, die steuerliche Handhabung der Geschäftsprozesse richtig im ERP-System abzubilden. Aus organisatorischer Sicht kann die Problemrelevanz wie folgt begründet werden:

- Die Arbeitsteilung in Organisationen, in denen ERP-Systeme eingesetzt werden, kann als hoch eingeschätzt werden. Dies bedeutet, dass einzelne Personen nicht mehr den vollständigen Überblick über den umsatzsteuerlich relevanten Geschäftsvorfall haben. Im Rahmen dieser Arbeitsteilung müssen sich die beteiligten Personen insofern auf das System und dessen Einstellungen bezüglich der umsatzsteuerlichen Einordnung von Geschäftsvorfällen verlassen. 
- Expandiert eine Unternehmung, so werden ggfs. neue Geschäftsvorfälle auftreten, deren umsatzsteuerliche Behandlung im System nicht anfangs vorgesehen war. Dies ist insbesondere der Fall, wenn grenzüberschreitende Geschäftsvorfälle relevant werden.

Aus rechtlicher Sicht kann eine besondere Problemrelevanz wie folgt abgeleitet werden:

- Wird durch eine Betriebsprüfung (Außenprüfung) festgestellt, dass Geschäftsvorfälle umsatzsteuerfrei behandelt wurden, obwohl diese steuerpflichtig sind, so rechnet der Fiskus den Steuerbetrag heraus, sodass Umsatzsteuer nachbezahlt werden muss. Hieraus ergeben sich erhebliche Liquiditätsrisiken.

- Wird vom Fiskus festgestellt, dass Geschäftsvorfälle umsatzsteuerlich falsch behandelt wurden und entsteht daraus ein höherer Steueranspruch, so muss zusätzlich zur Nachzahlung mit einer Verzinsung des höheren Steueranspruchs gerechnet werden. Die Zinsbeträge können sehr hoch sein, da Betriebsprüfungen erst Jahre nach der Steuerentstehung stattfinden können.

- Die Finanzverwaltung hat besonderes Augenmerk auf die Umsatzsteuer, da durch Vorsteuerbetrug dem Staat erhebliche Einkommenseinbußen entstehen. Insbesondere sog. Karussellgeschäfte (Missing Trader or Carousel Fraud, siehe Ainsworth 2006) haben zu dieser Aufmerksamkeit geführt.

- Die Finanzverwaltung kann die Umsatzteuer - im Gegensatz zu den anderen Steuerarten - spontan und ohne Anmeldung außerhalb einer Außenprüfung prüfen (sog. Umsatzsteuernachschau $\$ 27 b \mathrm{bStG}$ ).

\section{Umsatzsteuerliche Grundbegriffe und Definitionen}

\subsection{Grundlagen}

Das Umsatzsteuerrecht ist innerhalb der EU Mitgliedsstaaten - im Gegensatz zu den Ertragsteuern - weitgehend harmonisiert. Das Umsatzsteuerecht muss dem Grundprinzip des freien Warenverkehrs Rechnung tragen und wurde deshalb im Rahmen der Richtlinie 2006/112/EG vom 28. November 2006 (Mehrwertsteuersystemrichtlinie) geregelt. Die Mitgliedstaaten müssen die Richtlinie jeweils in nationales Recht umsetzen. Folgend werden zentrale umsatzsteuerlich relevante und zu unterscheidende Geschäftsvorfälle dargestellt. Dies dient lediglich einem generellen Einblick in die Regelungen des Umsatzsteuerrechts und es kann aufgrund der Rechtskomplexität kein Anspruch auf Vollständigkeit erhoben werden. Dabei wird sich auf die Lieferung beschränkt und nicht auf sonstige Leistungen eingegangen. Auch können spezielle Aspekte wie unentgeltliche Wertabgaben, Umkehr 
der Steuerschuld, Werklieferungen und -leistungen, Kommissionsgeschäfte, innergemeinschaftliche Dreiecksgeschäfte und spezielle Besteuerungsregelungen für Kraftwagen, Energie und Versandgeschäfte etc. nicht dargestellt werden.

Lieferungen und sonstige Leistungen: Das Umsatzsteuerrecht unterscheidet nur zwei Arten von Leistungen, die unterschiedlich gewürdigt werden müssen: Lieferungen und sonstige Leistungen. Eine Lieferung ist in der Regel die Verschaffung der Verfügungsmacht an einem Gegenstand (\$3 (1) UStG). Sonstige Leistungen werden schlicht negativ abgegrenzt: ,Sonstige Leistungen sind Leistungen, die keine Lieferungen sind“ (\$3 (9) UStG). Darunter fallen auch alle Dienstleistungen. Bei der Kategorisierung von Geschäftsvorfällen ist die Art der Leistung die erste festzustellende Eigenschaft.

Steuerbare Umsätze, Steuerfreiheit und Steuerpflicht: Das Umsatzsteuerrecht unterscheidet steuerbare, steuerfreie und steuerpflichtige Leistungen. Eine Leistung kann nur steuerfrei oder steuerpflichtig sein, wenn sie generell steuerbar ist. Steuerbarkeit bedeutet, dass der deutsche Fiskus generell ein Besteuerungsrecht ausüben könnte. Steuerbarkeit ist u.a. gegeben, wenn der Ort der Leistung im Inland ist. Aus system- oder sozialpolitischen Gründen können aber steuerbare Leistungen steuerfrei gestellt werden. Ein Beispiel für eine systemorientierte Steuerfreistellung ist die Ausfuhrlieferung, weil die ausländische Importnation in der Regel die Einfuhr steuerbar und steuerpflichtig behandeln wird und insofern eine Doppelbesteuerung vorliegen würde. Ein Beispiel für eine sozialpolitische Steuerfreiheit ist die Steuerfreiheit für Mieten oder ärztliche Behandlungen ( $\$ 4 \mathrm{UStG}$ ).

\subsection{Steuerlich relevante Geschäftsvorfälle}

Lieferungen und sonstige Leistungen im Inland. Vereinfachend gesagt, sind dies die „normalen“ Umsätze, die im Inland getätigt werden und die entweder steuerfrei sind oder bei denen 7\% bzw. 19\% USt erhoben wird: „... Lieferungen und sonstigen Leistungen, die ein Unternehmer im Inland gegen Entgelt im Rahmen seines Unternehmens ausführt.“" (\$1 (1) Nr.1 UStG).

Innergemeinschaftlicher Erwerb und Innergemeinschaftliche Lieferungen.

Ein innergemeinschaftlicher Erwerb ist im Inland steuerbar und steuerpflichtig. Ein gewöhnlicher innergemeinschaftlicher Erwerb liegt vor, wenn ein Gegenstand bei einer Lieferung an den Abnehmer (Erwerber) aus dem Gebiet eines Mitgliedstaates in das Gebiet eines anderen Mitgliedstaates gelangt. Der Erwerber ist dabei ein Unternehmer, der den Gegenstand für sein Unternehmen erwirbt und die Lieferung an den Erwerber wird durch einen Unternehmer gegen Entgelt im Rahmen seines Unternehmens ausgeführt. An dieser Stelle sei angemerkt, dass der Erwerber - nicht der Lieferer - die Steuer abzuführen hat und diese jedoch wieder als Vorsteuer abziehen kann, soweit er dazu berechtigt ist. Was für den Erwerber ein innergemeinschaftlicher Erwerb ist, ist für den Lieferer i.d.R. eine innergemeinschaftliche Lieferung. Die innergemeinschaftliche Lieferung ist steuerbar, aber steuerfrei ( $\$ 4 \mathrm{Nr} 1$. A, $\$ 6 a \mathrm{UStG})$. 
Innergemeinschaftliches Verbringen. Innergemeinschaftliches Verbringen ist eine Spielart des innergemeinschaftlichen Erwerbs: „Als innergemeinschaftlicher Erwerb gegen Entgelt gilt das Verbringen eines Gegenstands des Unternehmens aus dem übrigen Gemeinschaftsgebiet in das Inland durch einen Unternehmer zu seiner Verfügung“ ( $\$ 1$ a (2) UStG). Es handelt sich um eine gesetzliche Fiktion. Beim innergemeinschaftlichen Verbringen sind nicht zwei Geschäftspartner beteiligt. Es wird nur ein Gegenstand innerhalb eines Unternehmens zur weiteren Verwendung über eine EU-Grenze verbracht.

Einfuhr und Ausfuhrlieferung. Die Begriffe Einfuhr und Ausfuhrlieferung beziehen sich immer auf Warenbewegungen in Bezug auf das nicht-EU Ausland. Die Einfuhr von Gegenständen ins Inland ist steuerbar und i.d.R. steuerpflichtig ( $\$ 1(1)$ Nr. 5). Die Ausfuhr von Gegenständen hingegen ist auch steuerbar, jedoch steuerbefreit ( $\$ 4 \mathrm{Nr}$. 1a, \$6 UStG).

Reihengeschäft. Ein Reihengeschäft ist immer dann gegeben, wenn ein Gegenstand mehrmals verkauft wird, also umsatzsteuerlich mehrere Lieferungen vorliegen, jedoch der Gegenstand nur einmal tatsächlich physisch geliefert wird. Alle Geschäftsvorfälle aller Beteiligten müssen dann jedoch umsatzsteuerlich gewürdigt werden. Das Reihengeschäft wird besonders dann kompliziert, wenn die beteiligten Geschäftspartner in verschiedenen Ländern sitzen und der Gegenstand selbst auch über die Grenzen verbracht wird. Die Frage ist dann, in welchem Land jeweils die Umsatzversteuerung vorzunehmen ist (Ort der Lieferung). Die Formulierung im Gesetz lässt die Komplexität dieses Falls bereits erahnen: „Schließen mehrere Unternehmer über denselben Gegenstand Umsatzgeschäfte ab und gelangt dieser Gegenstand bei der Beförderung oder Versendung unmittelbar vom ersten Unternehmer an den letzten Abnehmer, ist die Beförderung oder Versendung des Gegenstands nur einer der Lieferungen zuzuordnen. Wird der Gegenstand der Lieferung dabei durch einen Abnehmer befördert oder versendet, der zugleich Lieferer ist, ist die Beförderung oder Versendung der Lieferung an ihn zuzuordnen, es sei denn, er weist nach, dass er den Gegenstand als Lieferer befördert oder versendet hat.“ (\$3 (6) S. 5 UStG regelt die sogenannte ,bewegte Lieferung“ = „Beförderung oder Versendung“ in der Kette). Der Ort der Lieferungen der anderen Unternehmer in der Kette (sog. ,ruhende Lieferungen") wird wie folgt geregelt ( $\$ 3$ (7) UStG): (1) Lieferungen, die der Beförderungs- oder Versendungslieferung vorangehen, gelten dort als ausgeführt, wo die Beförderung oder Versendung des Gegenstands beginnt. (2) Lieferungen, die der Beförderungs- oder Versendungslieferung folgen, gelten dort als ausgeführt, wo die Beförderung oder Versendung des Gegenstands endet.

Die umsatzsteuerliche Würdigung der Lieferung im Rahmen eines Reihengeschäfts und die damit verbundene Abbildung im ERP-System ist schwierig, da die Beurteilung der eigenen Lieferung nicht unabhängig von der Beurteilung der Lieferungen der anderen Unternehmer in der Reihe ist. Insbesondere hängt die Würdigung der Lieferungen auch von vereinbarten Incoterms innerhalb der Reihe ab, da 
diese einen Einfluss auf Kosten und Risiko der Versendung haben (R31a (10) UStR).

\section{Identifikation umsatzsteuerlicher Anforderungen bei der Einführung von ERP-Systemen}

Die Analyse der umsatzsteuerlichen Anforderungen bei der Einführung eines ERP-Systems sollte strukturiert stattfinden. Im Folgenden werden zwei alternative Methoden vorgestellt, wie aus den Vorschriften und Anforderungen des Umsatzsteuerrechts auf die technischen Einstellungen in einem System geschlossen werden kann:

1. Lineare Methode: Von der Rechtsquelle zu den Systemeinstellungen und -daten.

2. Entscheidungsbaummethode: Top-Down von den maßgeblichen Tatbestandmerkmalen zur Rechtsquelle und anschließend zu den Systemeinstellungen und -daten.

\section{Lineare Methode}

Bei der Anforderungsermittlung mit der linearen Methode wird immer mit der Rechtsquelle im Umsatzsteuerrecht begonnen. Im Rahmen der Anforderungserhebung für ein ERP-System muss also untersucht werden, ob die Tatbestandsmerkmale der Rechtsquelle von Geschäftsvorfällen der implementierenden Organisation erfüllt werden, um dann auf notwendige Einstellungen im System zu schließen. Die Lineare Methode verwendet für jede Rechtsquelle eine Sequenz von zehn Schritten.

Zur Anwendung der linearen Methode müssen alle relevanten Rechtsquellen bekannt und im Rahmen eines ERP-Implementierungsprojektes mit der beschriebenen Schrittfolge geprüft werden. An diesem Prozess nehmen idealerweise Steuer-, als auch ERP-Experten teil. Durch die dargestellte lineare Methode soll das Wissen der Experten geeignet integriert werden. 
Tabelle 1: Darstellung der linearen Methode

\begin{tabular}{|c|c|c|}
\hline $\mathrm{Nr}$ & Schritt & Beispiel \\
\hline 1 & $\begin{array}{l}\text { Rechtsquelle: Die für Geschäftsvorfälle relevan- } \\
\text { ten umsatzsteuerlichen Rechtsquellen werden } \\
\text { dem Gesetz entnommen, um die Relevanz in } \\
\text { dem Implementierungsprojekt zu prüfen. }\end{array}$ & $\begin{array}{l}\text { \$1 (1) Nr. } 5 \text { UStG i.v.m. \1a UStG Steuerbarkeit des } \\
\text { innergemeinschaftlichen Erwerbs. }\end{array}$ \\
\hline 2 & Der Gesetzestext wird notiert. & $\begin{array}{l}\text { „(1) Ein innergemeinschaftlicher Erwerb gegen Ent- } \\
\text { gelt liegt vor, wenn die folgenden Voraussetzungen } \\
\text { erfüllt sind: ..." }\end{array}$ \\
\hline 3 & $\begin{array}{l}\text { Die Tatbestandsmerkmale werden aus dem } \\
\text { Gesetzestext entnommen und zunächst } \\
\text { aufgelistet. }\end{array}$ & $\begin{array}{l}\text { Lieferung, Mitgliedstaat A, Mitgliedstaat B, Erwerber, } \\
\text { Unternehmer, Unternehmen, Lieferer, Unternehmer, } \\
\text { im Rahmen des Unternehmens, gegen Entgelt. }\end{array}$ \\
\hline 4 & $\begin{array}{l}\text { Die Tatbestandsmerkmale werden aufeinander } \\
\text { bezogen. Die Bedingungen der Tatbestands- } \\
\text { merkmale werden in einer Pseudoprogram- } \\
\text { miersprache notiert, um die Verknüpfung der } \\
\text { Tatbestandsmerkmale zu verdeutlichen. }\end{array}$ & 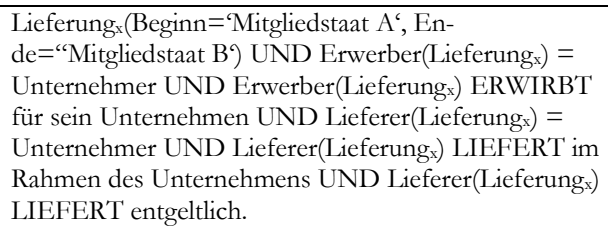 \\
\hline 5 & $\begin{array}{l}\text { Es wird geschlussfolgert, ob Geschäftsvorfälle } \\
\text { existieren, die die Tatbestandsmerkmale } \\
\text { erfüllen. }\end{array}$ & $\begin{array}{l}\text { Ja / Nein und daraus resultierende Rechtsfolge: } \\
\text { Steuerbar und steuerpflichtig im Inland, Vorsteuerab- } \\
\text { zugsberechtigt. }\end{array}$ \\
\hline 6 & $\begin{array}{l}\text { Es wird erhoben, welche Belege / Datenbestände } \\
\text { im ERP-System die Informationen zu den } \\
\text { relevanten Tatbestandmerkmalen enthalten. }\end{array}$ & $\begin{array}{l}\text { Kreditorenstammdaten, Lieferschein, Eingangsrech- } \\
\text { nung }\end{array}$ \\
\hline 7 & $\begin{array}{l}\text { Es werden die betroffenen Geschäftsprozesse } \\
\text { identifiziert }\end{array}$ & Beschaffung \\
\hline 8 & $\begin{array}{l}\text { Es werden die betroffenen technischen } \\
\text { Transaktionen (bei SAP, in anderen ERP- } \\
\text { Systemen ggfs. ein anderer Terminus) identi- } \\
\text { fiziert. }\end{array}$ & z. B. SAP: ME21, MIGO, MIRO \\
\hline 9 & $\begin{array}{l}\text { Es werden die betroffenen } \\
\text { Customizingeinstellungen ermittelt. }\end{array}$ & $\begin{array}{l}\text { z. B. SAP: IMG: Finanzwesen -> Grundeinstellungen } \\
\text { Finanzwesen -> Umsatzsteuer }->\text { Berechnung }-> \\
\text { Umsatzsteuerkennzeichen definieren }\end{array}$ \\
\hline 10 & $\begin{array}{l}\text { Es werden (bei Bedarf) betroffene Datentabel- } \\
\text { len und / oder Datenfelder ermittelt. }\end{array}$ & $\begin{array}{l}\text { z. B. SAP: BKPF (Belegköpfe), BSEG (Belegsegmen- } \\
\text { te) }\end{array}$ \\
\hline
\end{tabular}

\section{Entscheidungsbaummethode}

Die Entscheidungsbaummethode beginnt nicht mit der Rechtsquelle, sondern mit Tatbestandsmerkmalen, die dann zu relevanten Rechtsquellen führen. Sie geht gewissermaßen umkehrt im Vergleich zur linearen Methode vor. Dabei werden auf oberer Ebene Tatbestandsmerkmale abgeprüft, die geeignet sind, die verschiedenen vorhandenen umsatzsteuerlich zu würdigenden Geschäftsvorfälle geeignet zu trennen (siehe zu Entscheidungsbaumverfahren Chamoni 2006, S. 268). Der Entscheidungsbaum muss zunächst manuell von steuerlichen Experten aufgestellt werden und dann wird im Rahmen des ERP-Einführungsprojektes nachvollzogen, 
welche resultierenden Geschäftsvorfälle konkret zu berücksichtigen und was die umsatzsteuerlichen Folgen sind.

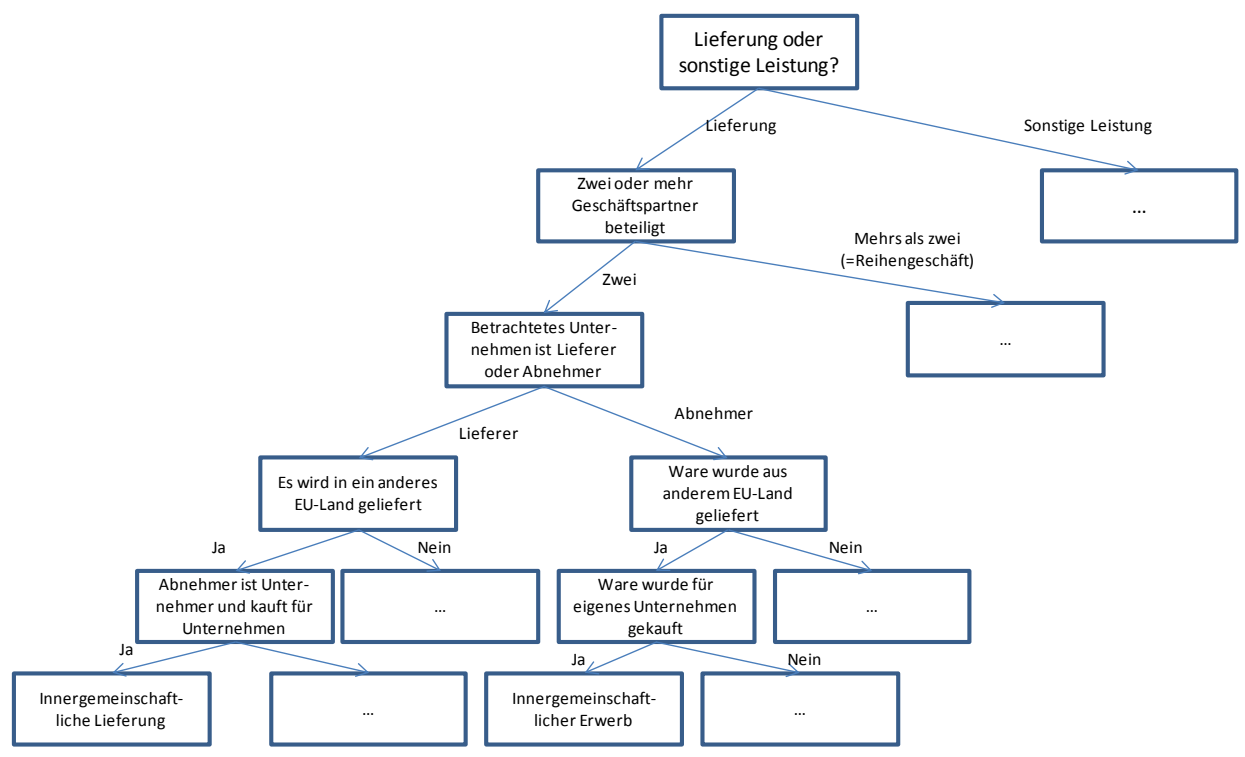

\section{Abbildung 1: Vereinfachter Entscheidungsbaum zur Einschätzung vorhandener Geschäftsvorfälle aus Sicht eines in Deutschland tätigen Unterneh mens}

Es ist deutlich, dass der Entscheidungsbaum erheblich komplex ist. Er kann deshalb nur in Ansätzen skizziert werden. Alle Verästelungen des Entscheidungsbaums sind während des ERP-Einführungsprozesses auf Relevanz zu prüfen. Die relevanten Tatbestandsmerkmale befinden sich hier jeweils auf den Kanten des Pfades zum Blatt. Wurde festgestellt, dass ein Blatt bezüglich der vorliegenden Geschäftsprozesse relevant ist, so kann mit den Schritten ab Schritt sechs der linearen Methode fortgesetzt werden. An dieser Stelle geht die Entscheidungsbaummethode in die lineare Methode über.

\section{VAT Compliance im Regelbetrieb von ERP-Systemen}

Der vorangegangene Abschnitt hat sich mit VAT Compliance Aspekten während einer ERP-Systemeinführung auseinandergesetzt. Oftmals ist jedoch auch von Interesse, ob alle Geschäftsvorfälle im laufenden Betrieb umsatzsteuerlich korrekt abgewickelt werden. Ggfs. kommen neue Geschäftsvorfälle während des Regelbetriebs hinzu, die einer umsatzsteuerlichen Abwicklung bedürfen, die bisher im System nicht vorgesehen wurden oder die nur mit manuellem Eingriff abgewickelt werden können. Dies kann z. B. sein, wenn die Geschäftstätigkeit vom Inland auf 
das Ausland expandiert. Im Folgenden wird eine Vorgehensweise für die Anwendung im Regelbetrieb skizziert, um festzustellen, ob die umsatzsteuerliche Abwicklung bereits stattgefundener Transaktionen ordnungsgemäß war. Die Vorgehensweise wird VAT-Scan genannt und ist darauf ausgelegt, durch Analyse betriebswirtschaftlicher Daten im ERP-System auf umsatzsteuerliche Probleme zu schließen. Die Vorgehensweise gibt - bei fachkundiger Interpretation der Ergebnisse nur Hinweise und kann deshalb nicht als eine exakte Methode verstanden werden. Theoretisch können natürlich auch die Analysemethoden des vorherigen Abschnitts eingesetzt werden (lineare oder Entscheidungsbaummethode), jedoch zielen diese nicht auf die automatische Analyse vorhandener Daten ab, sondern eher auf eine abstrakte Einordnung von Geschäftsvorfällen ohne Einbezug der tatsächlichen Datengrundlage im relevanten ERP-System. Ein VAT-Scan kann als eine Beratungsdienstleistung aufgefasst werden, hinter der eine definierte Vorgehensweise steht und die Beratungsaspekte des Steuerrechts mit Aspekten der ITbzw. ERP-System Beratung integriert.

\section{Ablauf eines VAT Scan}

Der Ablauf eines VAT Scan ist in mehrere sequentielle Phasen unterteilt. Die Einhaltung der Phasen dient dem strukturierten Erkenntnisgewinn. Vorgesehen sind die Phasen „Unternehmensumfeld aufklären“, „Analysebereich abgrenzen“, „Analyse von Stammdaten“, „Analyse von Bewegungsdaten“, „Risikoeinschätzung" und „Empfehlungen“.

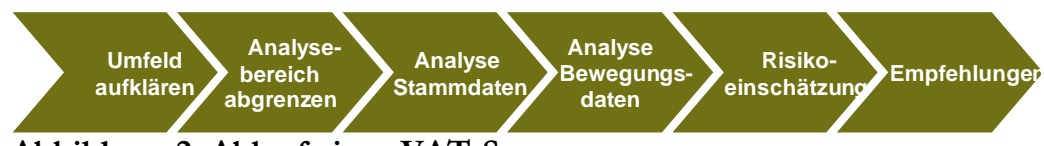

Abbildung 2: Ablauf eines VAT-Scan

In der Phase „Umfeld aufklären“ wird die Organisation des Unternehmens betrachtet. Es erfolgt eine Aufnahme des Umfelds bezüglich des ERP-Systems und der Geschäftsprozesse des Unternehmens. Es können bereits hier Schwerpunkte identifiziert werden, die im Rahmen des VAT-Scans besondere Beachtung erhalten sollten.

In der Phase „Analysebereich abgrenzen“ wird festgelegt, welche Daten untersucht werden sollen und welche Datenanalysen angewendet werden. Die Datenanalysen sind dabei derart vorkonfiguriert, dass diese Hinweise zur Beantwortung z. B. folgender Fragen geben können: „Wurden Rechnungen doppelt im System erfasst?", „Haben wir Lieferanten doppelt bezahlt?", „Sind unsere Kundendaten nicht gepflegt (z. B. USt-Identifikationsnummer)?“, „Liegen Redundanzen in den Kreditorenstammdaten vor (z. B. gleiche USt-Identifikationsnummer wird bei mehreren Kreditoren verwendet)?“ 
Es folgen zwei Phasen, in der die Analysen durchgeführt werden. Diese Analysephasen wurden gentrennt nach Analysen im Bereich von Stammdaten und Analysen im Bereich von Bewegungsdaten. Die Ergebnisse der Analysen können im Rahmen des VAT-Scans als Indikatoren bezeichnet werden. Die Ergebnisse lassen nicht zwingend automatisch Rückschluss auf umsatzsteuerliche Probleme zu, sondern es ist stets die Würdigung der Analyseergebnisse durch einen Fachkundigen notwendig.

In der anschließenden Phase werden die Indikatoren interpretiert und umsatzsteuerliche Risiken abgeleitet. Einerseits muss hier abgeschätzt werden, welches Fehlerpotenzial insgesamt in den Daten der Geschäftsprozesse des Unternehmens stecken und andererseits ist die Abschätzung des Entdeckungsrisikos durch Betriebsprüfungen notwendig.

Die letzte Phase leitet aus den Risiken Handlungsempfehlungen ab. Die Handlungsempfehlungen können darauf abzielen (1) vergangene Geschäftsvorfälle zu korrigieren, (2) zukünftige Geschäftsvorfälle richtig abzuwickeln oder (3) weiteren Analysebedarf aufzuzeigen.

Die folgende Abbildung zeigt, welche Aspekte in den verschiedenen Phasen berücksichtigt werden müssen. Mögliche Fehlerquellen werden in den Phasen „Umfeld aufklären“ identifiziert. Die zu verwendenden Analysen bzw. Indikatoren werden in den Phasen „Analysebereich abgrenzen“ und „Analyse Stammdaten“ sowie „Analyse Bewegungsdaten“ relevant. Der Untersuchungsbereich in Bezug auf die relevanten Geschäftsprozesse wird in der Phase „Analysebereich abgrenzen" festgelegt.

\begin{tabular}{|c|c|c|}
\hline Fehlerquellen & Indikatoren & Untersuchungsbereich \\
\hline Steuersatz & \multirow{3}{*}{$\begin{array}{l}\text { SCAN Eingangsteuer } \\
: \text { Eingangs Erechnungen vor Periodenende } \\
: \text { Umsatzserlose ohne USt (Inland) } \\
\text { Skonto / Rababtt/ Preisnachlass }\end{array}$} & Einkauf \\
\hline Manuelle Korrekturen & & Dokumentenverarbeitung \\
\hline $\begin{array}{l}\text { Unberücksichtigte } \\
\text { Transaktionen }\end{array}$ & & Umsatzsteuer- \\
\hline Fehlende Voraussetzung & \multirow{2}{*}{ 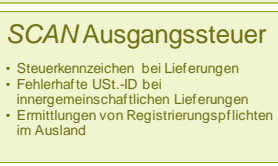 } & Personal \\
\hline Systemfehler & & Verkauf \\
\hline Abstimmung & \multirow{3}{*}{$\begin{array}{l}\text { SCAN Finanzwesen } \\
\text { - Plausibilitätspruntungen } \\
\text { 2.B. vorkommende Steuerkategorien je } \\
\text { Sachkonto } \\
\text { : Innenumsatze } \\
\text { Zeititunkt der Umsatzsteuerbuchung - } \\
\text { Vergleich Buchungs- und Belegdatum }\end{array}$} & Anlagevermögen \\
\hline Ausweisfehler & & Rechnungswesen \\
\hline$\cdots \cdots$ & & Customizing System \\
\hline
\end{tabular}

\section{Abbildung 3: Zu berücksichtigende Aspekte beim VAT-Scan}

Die Indikatoren geben Hinweise auf umsatzsteuerliche Problembereiche. Die Indikatoren sind dabei Rechtsvorschriften oder generell bekannten umsatzsteuerlichen Problemen zugeordnet, um bei der Schlussfolgerung und Interpretation der Ergebnisse die möglichen Problemkreise einzugrenzen. 
Tabelle 2: Zuordnung von Indikatoren zu umsatzsteuerlichen Problemkreisen

\begin{tabular}{|l|l|l|}
\hline \multicolumn{1}{|c|}{ Indikator } & \multicolumn{1}{|c|}{ Beschreibung } & $\begin{array}{c}\text { Zuordnung zu umatz- } \\
\text { teuerlichem Problem- } \\
\text { feld }\end{array}$ \\
\hline $\begin{array}{l}\text { Steuerkennzeichen } \\
\text { ausländischer Lieferanten }\end{array}$ & $\begin{array}{l}\text { Untersuchung der verwendeten Steuerkennzeichen } \\
\text { aller Eingangsrechnungen ausländischer Lieferanten } \\
\text { (mit Kennzeichen ob es sich um ein EU- oder } \\
\text { Drittland handelt) }\end{array}$ & $\begin{array}{l}\text { Innergemeinschaftlicher } \\
\text { Erwerb }\end{array}$ \\
\hline $\begin{array}{l}\text { Lieferanten mit verschie- } \\
\text { denen Steuerkennzeichen }\end{array}$ & $\begin{array}{l}\text { Untersuchung der Lieferanten, zu denen Rechnun- } \\
\text { gen mit verschiedenen Steuerkennzeichen erstellt } \\
\text { worden sind }\end{array}$ & $\begin{array}{l}\text { Innergemeinschaftlicher } \\
\text { Erwerb }\end{array}$ \\
\hline $\begin{array}{l}\text { Untersuchung verwende- } \\
\text { ter Steuerkennzeichen }\end{array}$ & $\begin{array}{l}\text { Untersuchung der verwendeten Steuerkennzeichen } \\
\text { aller Eingangsrechnungen mit Einzelpostenausgabe } \\
\text { und Ermittlung der dazugehörigen Einkaufsbelege. }\end{array}$ & $\begin{array}{l}\text { Innergemeinschaftlicher } \\
\text { Erwerb, Reihen- } \\
\text { Dreiecksgeschäfte }\end{array}$ \\
\hline $\begin{array}{l}\text { Untersuchung der nicht } \\
\text { abgezogenen Vorsteuer }\end{array}$ & $\begin{array}{l}\text { Untersuchung aller Eingangsrechnungen, die ohne } \\
\text { Vorsteuer gebucht worden sind }\end{array}$ & \begin{tabular}{l} 
Rechnungskorrekturen \\
\hline
\end{tabular} \\
\hline
\end{tabular}

\section{$7 \quad$ Fazit}

Der vorliegende Beitrag hat Vorgehensweisen skizziert, wie umsatzsteuerliche Regelungen bei der Einführung und während des Betriebs eines ERP-Systems berücksichtigt werden können. Im Rahmen der Einführung eines ERP-Systems wurden zwei Methoden vorgestellt, die lineare Methode und das Entscheidungsbaumverfahren. Da das Entscheidungsbaumverfahren isoliert an einzelne Tatbestandsmerkmale anknüpft, kann vermutet werden, dass dieses für NichtSteuerexperten die einfachere Methode ist, da die lineare Methode die Kenntnis einer ganzen Rechtsnorm bereits voraussetzt. Für den Regelbetrieb wurde die VAT-Scan Vorgehensweise vorgestellt, die keine exakte Vorgehensweise ist, jedoch einen Einblick in die umsatzsteuerlichen Verhältnisse innerhalb eines ERP-Systems in relativ kurzer Zeit erlauben soll. Als weiterer Forschungsbedarf wird insbesondere die Anwendung der beschriebenen Vorgehensweisen gesehen. Für den VATScan liegen bereits Ergebnisse aus einer Anwendung vor, die jedoch noch nicht verallgemeinert werden können. Zukünftige Anwendungsfälle werden die Vorgehensweise weiter verfeinern.

\section{Literatur}

Ainsworth, RT (2006) Carousel Fraud in the EU: A Digital VAT Solution. Tax Notes International, p. 443, May 1, 2006; Boston Univ. School of Law Working Paper No. 06-23.

Biagioli C et al. (2005) Automatic Semantics Extraction in Law Documents,

Proceedings of the 10th International Conference on AI and Law, pp. 133-140, June 2005. 
Breaux TD, Vail MW, Antón AI (2006) Towards Regulatory Compliance:

Extracting Rights and Obligations to Align Requirements with Regulations, Proceedings of the 13th IEEE International Conference on Requirements Engeering, pp. 46-55, September 2006.

Breaux TD, Antón AI (2008a) Analyzing Regulatory Rules for Privacy and Security Requirements, IEEE Transactions on Software Engineering, Vol.34, No1, pp.5-20, Jan/Feb 2008.

Breaux TD, Antón AI, Boucher K, Dorfman M (2008b) Legal requirements, compliance and practice: An industry case study in accessibility, Proceedings of IEEE 16th International Requirements Engineering Conference (RE’08), pp. 43-52, September 2008.

Chamoni, P (2006) Analytische Informationssysteme. Business-IntelligenceTechnologien und -Anwendungen, 3., vollst. überarb. Aufl. Berlin: Springer.

Ghanavati S, Amyot D, Peyton L (2007) A Requirements Management Framework for Privacy Compliance, Proceedings of the 10th Workshop on Requirements Engineering (WER'07), pp. 149-159, Canada, May 2007.

Goossenaerts JBM., Zegers A., Smits JM (2009) A Multi-Level Model-Driven

Regime for Value-Added Tax Compliance in ERP Systems (March 23, 2009).

Computers in Industry, Forthcoming.

Karagiannis D (2008) A Business Process-Based Modelling Extension for

Regulatory Compliance, Proceedings of Multikonferenz Wirtschaftsinformatik, MKWI 2008, pp. 1159- 1173, February 2008.

Kiavitskaya N, Krausova A, Zannone N (2008): Why Eliciting and Managing Legal Requirements is Hard", 1st Int. Workshop on Requirements Engineering and Law, In conj. with the 16th IEEE International Requirements Engineering Conference, 2008.

Kiyavitskaya N, Zeni N, Breaux TD, Antón AI, Cordy JR., Mich L, Mylopoulos J (2007) Extracting Rights and Obligations from Regulations: Toward a ToolSupported Process, Proceedings of the 22nd IEEE/ACM International Conference on Automated Software Engineering (ASE '07), pp.429-432, November 2007.

Maliszewski M (2008) Software zur Analyse steuerrelevanter Daten. Evaluation ausgewählter Softwaretools für die Vorbereitung auf eine elektronische Betriebsprüfung: VDM Verlag.

Nuseibeh B, Easterbrook S (2000) Requirements engineering: a roadmap. In: Proceedings of the Conference on The Future of Software Engineering. Limerick, Ireland: ACM, S. 35-46. 
Odenthal, R (2006) Prüfsoftware im Einsatz. Handbuch für die praktische Analyse von Unternehmensdaten: DATEV.

Otto PN, Antón AI (2007) Addressing Legal Requirements in Requirements Engineering, 15th IEEE Internationl Requirements Engineering Conference, pp.5-14, October 2007.

Palmirani M, Brighi R, Massini M (2003) Automated Extraction of Normative References in Legal Texts, Proceedings of the 9th International Conference on AI and Law, pp. 105-106, June 2003.

Siena A, Mylopoulos J (2008) From Laws to Requirements, 1st Int. Workshop on Requirements Engineering and Law, 2008. 